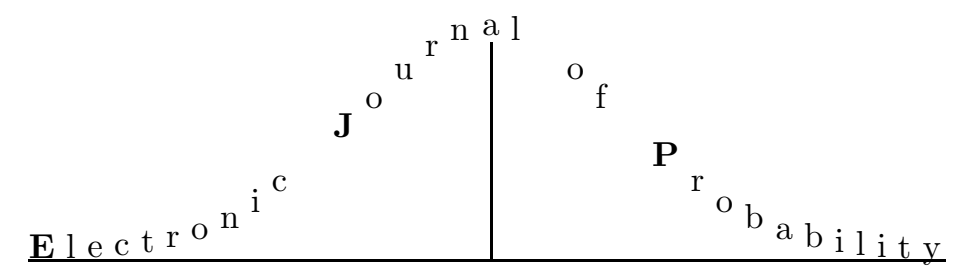

Vol. 11 (2006), Paper no. 45, pages 1184-1203.

Journal URL

http://www.math. washington.edu/ ejpecp/

\title{
Asymptotic behaviour of the simple random walk on the 2-dimensional comb *
}

\author{
Daniela Bertacchi \\ Università di Milano-Bicocca \\ Dipartimento di Matematica e Applicazioni \\ Via Cozzi 53, 20125 Milano, Italy \\ daniela.bertacchi@unimib.it
}

\begin{abstract}
We analyze the differences between the horizontal and the vertical component of the simple random walk on the 2 -dimensional comb. In particular we evaluate by combinatorial methods the asymptotic behaviour of the expected value of the distance from the origin, the maximal deviation and the maximal span in $n$ steps, proving that for all these quantities the order is $n^{1 / 4}$ for the horizontal projection and $n^{1 / 2}$ for the vertical one (the exact constants are determined). Then we rescale the two projections of the random walk dividing by $n^{1 / 4}$ and $n^{1 / 2}$ the horizontal and vertical ones, respectively. The limit process is obtained. With similar techniques the walk dimension is determined, showing that the Einstein relation between the fractal, spectral and walk dimensions does not hold on the comb
\end{abstract}

Key words: Random Walk, Maximal Excursion, Generating Function, Comb, Brownian Motion

AMS 2000 Subject Classification: Primary 60J10,05A15,60J65.

Submitted to EJP on February 15 2006, final version accepted October 302006.

\footnotetext{
*Research partly supported by Italian 2004 PRIN project "CAMPI ALEATORI"
} 


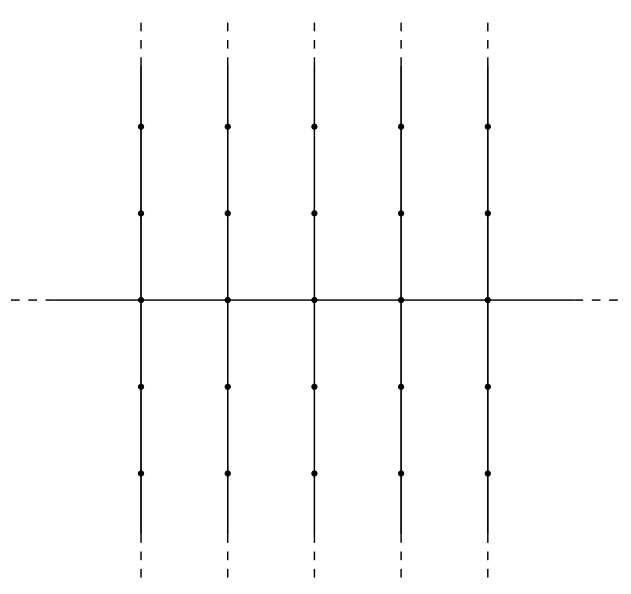

Figure 1: The 2-dimensional comb.

\section{Introduction and main results}

The 2-dimensional comb $\mathbf{C}_{2}$ is maybe the simplest example of inhomogeneous graph. It is obtained from $\mathbb{Z}^{2}$ by removing all horizontal edges off the $x$-axis (see Figure 1). Many features of the simple random walk on this graph has been matter of former investigations. Local limit theorems were first obtained by Weiss and Havlin (19) and then extended to higher dimensions by Gerl (10) and Cassi and Regina (4). More recently, Krishnapur and Peres (14) have shown that on $\mathbf{C}_{2}$ two independent walkers meet only finitely many times almost surely. This result, together with the space-time asymptotic estimates obtained in (3) for the $n$-step transition probabilities, suggests that the walker spends most of the time on some tooth of the comb, that is moving along the vertical direction. Indeed in (3, Section 10), it has been remarked that, if $k / n$ goes to zero with a certain speed, then $p^{(2 n)}((2 k, 0),(0,0)) / p^{(2 n)}((0,2 k),(0,0)) \stackrel{n \rightarrow \infty}{\longrightarrow} 0$.

Moreover the results in (3) imply that there are no sub-Gaussian estimate of the transition probabilities on $\mathbf{C}_{2}$. Such estimates have been found on many graphs: by Jones (12) on the 2-dimensional Sierpiński graph, by Barlow and Bass (1) on the graphical Sierpiński carpet and on rather general graphs by Grigor'yan and Telcs $((11 ; 18))$. These estimates involve three exponents which are usually associated to infinite graphs: the spectral dimension $\delta_{s}$ (which is by definition twice the exponent of $n^{-1}$ in local limit theorems), the fractal dimension $\delta_{f}$ (which is the growth exponent) and the walk dimension $\delta_{w}$ (by definition the expected time until the first exit from the $n$-ball around the starting point is of order $\left.n^{\delta_{w}}\right)$. These dimensions are in typical cases linked by the so-called Einstein relation: $\delta_{s} \delta_{w}=2 \delta_{f}$ (see Telcs $(16 ;$; 17)). The first two dimensions are known for $\mathbf{C}_{2}: \delta_{s}=3 / 2$ and $\delta_{f}=2$. In this paper we compute $\delta_{w}=2$, thus showing that the relation does not hold for this graph.

In order to point out the different behaviour of the random walk along the two directions we analyze the asymptotic behaviour of the expected values of the horizontal and vertical distances reached by the walker in $n$ steps. Different concepts of distance are considered: position after $n$ steps, maximal deviation and maximal span (see Section 2 for the definitions).

Studying the projections of the random walk onto the two axes one observes that the vertical projection is the simple random walk on $\mathbb{Z}$ with the addition of a loop at 0 . It is known that 
the expected values of the "distances" (i.e. position, maximal deviation and maximal span) in $n$ steps for the simple random walk on $\mathbb{Z}$ are of order $n^{1 / 2}$ (the estimates for the maximal deviation and maximal span were obtained by combinatorial methods by Panny and Prodinger in (15)). By comparison it is clear that the asymptotics coincide (to leading order) with the corresponding estimates for the vertical projection of the simple random walk on $\mathbf{C}_{2}$. We sketch the ideas of the proof at the end of Section 2 .

The behaviour of the horizontal projection of the random walk is less obvious: in Sections 3 4 and 5 we prove that the expected values of the horizontal distance, of its maximal deviation and of its maximal span after $n$ steps are all of order $n^{1 / 4}$ (the exact constants are determined in Theorem 3.1. Theorem 4.4 and Theorem 5.1 respectively).

The proofs are based on a Darboux type transfer theorem: we refer to (9, Corollary 2), but one may also refer to (2) and to the Hardy-Littlewood-Karamata theorem (see for instance (8)). This theorem (as far as we are concerned) claims that if

$$
F(z):=\sum_{n=0}^{\infty} a_{n} z^{n} \stackrel{z \rightarrow 1^{-}}{\sim} \frac{C}{(1-z)^{\alpha}}, \quad \alpha \notin\{0,-1,-2, \ldots\},
$$

and $F(z)$ is analytic in some domain, with the exception of $z=1$, then

$$
a_{n} \stackrel{n \rightarrow \infty}{\sim} \frac{C}{\Gamma(\alpha)} n^{\alpha-1}
$$

(by $a_{n} \sim b_{n}$ we mean that $a_{n} / b_{n} \stackrel{n \rightarrow \infty}{\rightarrow} 1$ ). Hence the aim of our computation is to determine an explicit expression of the generating functions of the sequences of expected values of the random variables we are interested in. This is done employing the combinatorial methods used by Panny and Prodinger in (15).

Since the notions of maximal deviation in $n$ steps and of first exit time from a $k$-ball are closely related, we determine the walk dimension of $\mathbf{C}_{2}$ in Section 4 . This is again done by comparison with the simple random walk on $\mathbb{Z}$.

In Section 6 we deal with the limit of the process obtained dividing by $n^{1 / 4}$ and by $n^{1 / 2}$ respectively the continuous time interpolation of the horizontal and vertical projections of the position after $n$ steps. As one would expect the limit of the vertical component is the Brownian motion, while the limit of the horizontal component is less obvious (it is a Brownian motion indexed by the local time at 0 of the vertical component). This scaling limit is determined in Theorem 6.1 Finally, Section $[$ is devoted to a discussion of the results, remarks and open questions.

\section{Preliminaries and vertical estimates}

The simple random walk on a graph is a sequence of random variables $\left\{S_{n}\right\}_{n \geq 0}$, where $S_{n}$ represents the position of the walker at time $n$, and if $x$ and $y$ are vertices which are neighbours then

$$
p(x, y):=\mathbb{P}\left(S_{n+1}=y \mid S_{n}=x\right)=\frac{1}{\operatorname{deg}(x)},
$$

where $\operatorname{deg}(x)$ is the number of neighbours of $x$, otherwise $p(x, y)=0$. In particular on $\mathbf{C}_{2}$ the non-zero transition probabilities $p(x, y)$ are equal to $1 / 4$ if $x$ is on the horizontal axis, and they are equal to $1 / 2$ otherwise. 
Given $x, y \in \mathbf{C}_{2}$, let

$$
p^{(n)}(x, y):=\mathbb{P}\left(S_{n}=y \mid S_{0}=x\right), \quad n \geq 0,
$$

be the $n$-step transition probability from $x$ to $y$ and $\mathbb{P}\left(S_{0}=(0,0)\right)=1$. Recall that the generating function of a sequence $\left\{a_{n}\right\}_{n \geq 0}$ is the power series $\sum_{n \geq 0} a_{n} z^{n}$; by definition the Green function associated to the random walk on a graph $X$ is the family of generating functions of the sequences $\left\{p^{(n)}(x, y)\right\}_{n \geq 0}, x, y \in X$, that is

$$
G(x, y \mid z)=\sum_{n \geq 0} p^{(n)}(x, y) z^{n} .
$$

The Green function, with $x=(0,0)$, on $\mathbf{C}_{2}$ can be written explicitly as (see (3) )

$$
G((0,0),(k, l) \mid z)= \begin{cases}\frac{1}{2} G(z)\left(F_{1}(z)\right)^{|k|}\left(F_{2}(z)\right)^{|l|}, & \text { if } l \neq 0 \\ G(z)\left(F_{1}(z)\right)^{|k|}, & \text { if } l=0\end{cases}
$$

where

$$
\begin{aligned}
G(z) & =\frac{\sqrt{2}}{\sqrt{1-z^{2}+\sqrt{1-z^{2}}}} ; \\
F_{1}(z) & =\frac{1+\sqrt{1-z^{2}}-\sqrt{2} \sqrt{1-z^{2}+\sqrt{1-z^{2}}}}{z} ; \\
F_{2}(z) & =\frac{1-\sqrt{1-z^{2}}}{z} .
\end{aligned}
$$

We refer to (21, Section 1.1) for more details on the random walks on graphs, transition probabilities and generating functions.

An interesting feature of a random walk is the distance reached in $n$ steps. This distance may be defined as $\left\|S_{n}\right\|$, the distance at time $n$ from the starting point, $\|\cdot\|$ denoting a suitable norm. On $\mathbf{C}_{2}$ the choice is between the usual distance on the graph $\|(x, y)\|_{1}=|x|+|y|$ and $\|(x, y)\|_{\infty}=\max \{|x|,|y|\}$.

Other "distances" are the maximal deviation and the maximal span in $n$ steps.

Definition 2.1. [a.]

1. The maximal deviation in $n$ steps is defined as

$$
D_{n}:=\max \left\{\left\|S_{i}\right\|: 0 \leq i \leq n\right\} .
$$

2. The maximal span in $n$ steps is defined as

$$
M_{n}:=\max \left\{\left\|S_{i}-S_{j}\right\|: 0 \leq i, j \leq n\right\} .
$$

When we need to stress the use of a particular norm $\|\cdot\|_{+}$, we use the notations $D_{n}\left(\|\cdot\|_{+}\right)$ and $M_{n}\left(\|\cdot\|_{+}\right)$. In order to deal with the two components of the walk, we write $S_{n}=\left(S_{n}^{x}, S_{n}^{y}\right)$ and benote by $D_{n}^{x}$ and $D_{n}^{y}$ the maximal deviations of the two processes $\left\{S_{n}^{x}\right\}_{n \geq 0}$ and $\left\{S_{n}^{y}\right\}_{n \geq 0}$ (defined on $\mathbb{Z}$ ) respectively. By $M_{n}^{x}$ and $M_{n}^{y}$ we denote the corresponding maximal deviations. 


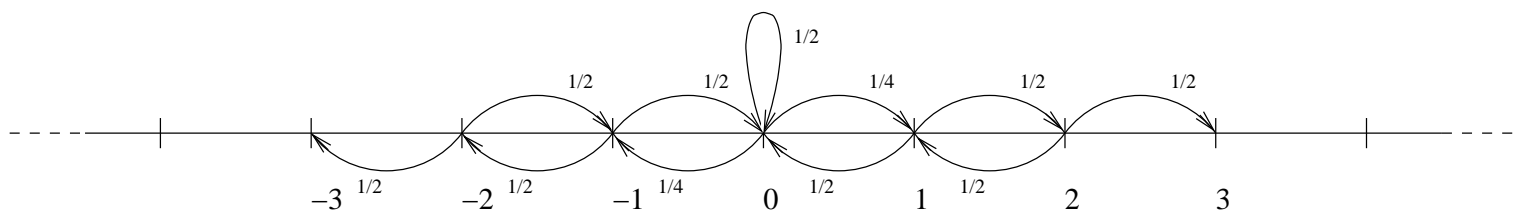

Figure 2: The vertical component of the simple random walk on $\mathbf{C}_{2}$.

Moreover we denote by $|\cdot|$ the usual norm on $\mathbb{Z}$. It is worth noting that on $\mathbb{Z}$ the maximal span may be equivalently defined as $\max \left\{S_{i}-S_{j}: 0 \leq i, j \leq n\right\}$.

Among the issues of this paper are the asymptotics of $\mathbb{E}\left(\left|S_{n}^{*}\right|\right), \mathbb{E}\left(D_{n}^{*}\right)$ and $\mathbb{E}\left(M_{n}^{*}\right)$, where $*$ is either $x$ or $y$.

Let us note that the asymptotics of the vertical component are already determined since they coincide (to leading order) with the corresponding estimates for the simple random walk on $\mathbb{Z}$. Indeed the vertical component of the simple random walk on $\mathbf{C}_{2}$ (represented in Figure 2) differs from the simple random walk on $\mathbb{Z}$ only in the presence of the loop at 0 . Hence we may associate to $\left\{S_{n}^{y}\right\}_{n \geq 0}$ two sequences of random variables: $\left\{Y_{n}^{\prime}\right\}_{n \geq 0}$ and $\left\{L_{n}\right\}_{n \geq 0}$, such that $\left\{Y_{n}^{\prime}\right\}_{n \geq 0}$ is a simple random walk on $\mathbb{Z}$, and $L_{n}$ is the number of loops performed by $\left\{S_{n}^{y}\right\}_{n \geq 0}$ up to time $n$. Thus $S_{n}^{y}=Y_{n}^{\prime}-E_{n}$, where the error term $E_{n}$ is the (random) sum of $L_{n}$ iid random variables $Z_{i}$ with $\mathbb{P}\left(Z_{i}= \pm 1\right)=\frac{1}{2}$. One easily proves that $E_{n}$ is negligible in all three estimates (for more details about $Y_{n}^{\prime}$ and $L_{n}$ see Section (6).

For the simple random walk $Y_{n}^{\prime}$ on $\mathbb{Z}, \mathbb{E}\left[\left|Y_{n}^{\prime}\right|\right] \sim \sqrt{2 / \pi} n^{1 / 2}$ may be obtained from the generating function of the walk using the technique of Theorem 3.1] while the asymptotics of its maximal deviation, $\sqrt{\pi / 2} n^{1 / 2}$, and of its maximal span, $\sqrt{8 / \pi} n^{1 / 2}$, may be found in (15) (Theorem 2.14 and Theorem 3.4 respectively). Thus the following result is obvious.

Theorem 2.2. [a.]

1. $\mathbb{E}\left[\left|S_{n}^{y}\right|\right] \stackrel{n \rightarrow \infty}{\sim} \sqrt{\frac{2}{\pi}} n^{1 / 2}$

2. $\mathbb{E}\left[D_{n}^{y}\right] \stackrel{n \rightarrow \infty}{\sim} \sqrt{\frac{\pi}{2}} n^{1 / 2}$;

3. $\mathbb{E}\left[M_{n}^{y}\right] \stackrel{n \rightarrow \infty}{\sim} \sqrt{\frac{8}{\pi}} n^{1 / 2}$.

\section{Mean distance}

The estimate of $\mathbb{E}\left[\left|S_{n}^{x}\right|\right]$ is quite easy and may be considered as a warm-up for the use of generating functions techniques.

Theorem 3.1.

$$
\mathbb{E}\left[\left|S_{n}^{x}\right|\right] \stackrel{n \rightarrow \infty}{\sim} \frac{1}{2^{3 / 4} \Gamma(5 / 4)} n^{1 / 4} .
$$

Proof. Since for $k \neq 0$,

$$
\mathbb{P}\left(\left|S_{n}^{x}\right|=k\right)=2 \sum_{l \in \mathbb{Z}} p^{(n)}((0,0),(k, l)),
$$


it is clear that (exchanging the order of summation)

$$
\sum_{n=0}^{\infty} \mathbb{E}\left[\left|S_{n}^{x}\right|\right] z^{n}=2 \sum_{k=1}^{\infty} k \sum_{l \in \mathbb{Z}} G((0,0),(k, l) \mid z) .
$$

By elementary computation one obtains

$$
\begin{aligned}
\sum_{n=0}^{\infty} \mathbb{E}\left[\left|S_{n}^{x}\right|\right] z^{n} & =G(z) \frac{F_{1}(z)}{\left(1-F_{1}(z)\right)^{2}} \frac{1}{1-F_{2}(z)} \\
& \underset{z \rightarrow 1^{-}}{\sim} \frac{1}{2^{3 / 4}(1-z)^{5 / 4}} .
\end{aligned}
$$

Thus, applying (9, Corollary 2) we obtain the claim.

The following Corollary is now trivial.

Corollary 3.2. On $\mathbf{C}_{2}, \mathbb{E}\left[\left\|S_{n}\right\|_{1}\right]$ and $\mathbb{E}\left[\left\|S_{n}\right\|_{\infty}\right]$ are both asymptotic, as $n$ goes to infinity, to $\sqrt{\frac{2}{\pi}} n^{1 / 2}$

\section{Mean maximal deviation and walk dimension}

\subsection{Maximal horizontal deviation}

In order to compute the generating function of $\left\{\mathbb{E}\left[D_{n}^{x}\right]\right\}_{n \geq 0}$, we first need an expression for another generating function.

Lemma 4.1. Let $h \in \mathbb{N}, l \in\{0, \ldots, h\}$. The generating function of the sequence $\left\{\mathbb{P}\left(D_{n}^{x} \leq h, S_{n}^{x}=l\right)\right\}_{n \geq 0}$ is

$$
\psi_{h, l}\left(\frac{1-\sqrt{1-z^{2}}}{2 z}\right) \cdot \frac{2\left(1-\sqrt{1-z^{2}}\right)}{z\left(\sqrt{1-z^{2}}-1+z\right)},
$$

where $\psi_{h, l}(z)$ is the generating function of the number of paths on $\mathbb{Z}$ of length $n$, from 0 to $l$ with maximal deviation less or equal to $h$.

Proof. Note that the paths we are interested in have no bound on the vertical excursions. Thus we may decompose the walk into its horizontal and vertical components, and consider each horizontal step as a vertical excursion (whose length might be zero) coming back to the origin plus a step along the horizontal direction.

Keeping this decomposition in mind, it is clear that the generating function of the sequence $\left\{\mathbb{P}\left(D_{n}^{x} \leq h, S_{n}=(l, 0)\right)\right\}_{n \geq 0}$ is

$$
\psi_{h, l}\left(\frac{z \widetilde{G}(0,0 \mid z)}{4}\right),
$$

where $\widetilde{G}(0, j \mid z)$ is the generating function of the probabilities of the $n$-step excursions along one single tooth of the comb (that is paths in Figure 2 which do not use the loop at zero), from $(x, 0)$ to $(x, j)$. 
Moreover we must admit a final excursion from $(l, 0)$ to $(l, j)$ for some $j \in \mathbb{Z}$, that is we must multiply by

$$
E(z):=\widetilde{G}(0,0 \mid z)+2 \sum_{j=1}^{\infty} \widetilde{G}(0, j \mid z)
$$

and the generating function of $\left\{\mathbb{P}\left(D_{n}^{x} \leq h, S_{n}^{x}=l\right)\right\}_{n \geq 0}$ is

$$
\psi_{h, l}\left(\frac{z \widetilde{G}(0,0 \mid z)}{4}\right) \cdot E(z) .
$$

Using (21, Lemma 1.13) and reversibility (see (21, Section 1.2.A)), it is not difficult to compute

$$
\begin{aligned}
& \widetilde{G}(0,0 \mid z)=\frac{2\left(1-\sqrt{1-z^{2}}\right)}{z^{2}}, \\
& \widetilde{G}(0, j \mid z)=\frac{1}{2} \widetilde{G}(0,0 \mid z)\left(\frac{1-\sqrt{1-z^{2}}}{z}\right)^{j}, \quad j \neq 0 .
\end{aligned}
$$

The claim is obtained noting that

$$
\begin{aligned}
\frac{z \widetilde{G}(0,0 \mid z)}{4} & =\frac{1-\sqrt{1-z^{2}}}{2 z} \\
E(z) & =\frac{2\left(1-\sqrt{1-z^{2}}\right)}{z\left(\sqrt{1-z^{2}}-1+z\right)} .
\end{aligned}
$$

\section{Proposition 4.2.}

$$
\sum_{n=0}^{\infty} \mathbb{E}\left[D_{n}^{x}\right] z^{n}=2 \frac{1+6 v^{2}+v^{4}}{(1-v)^{4}} \sum_{h \geq 1} \frac{v^{h}}{1+v^{2 h}}
$$

where $v$ is such that

$$
\frac{v}{1+v^{2}}=\frac{1-\sqrt{1-z^{2}}}{2 z}
$$

Proof. Let $\psi_{h, l}(z)$ be as in Lemma 4.1 and put $\psi_{h}(z)=\sum_{|l| \leq h} \psi_{h, l}(z)$. Then the generating function of $\left\{\mathbb{P}\left(D_{n}^{x} \leq h\right)\right\}_{n \geq 0}$ is

$$
H_{h}(z):=\psi_{h}\left(\frac{1-\sqrt{1-z^{2}}}{2 z}\right) \cdot \frac{2\left(1-\sqrt{1-z^{2}}\right)}{z\left(\sqrt{1-z^{2}}-1+z\right)}
$$

Thus we may write the generating function of $\mathbb{E}\left[D_{n}^{x}\right]$ as

$$
\sum_{n=0}^{\infty}\left(\sum_{h=0}^{\infty} \mathbb{P}\left(D_{n}^{x}>h\right)\right) z^{n}=\sum_{h=0}^{\infty}\left(\frac{1}{1-z}-H_{h}(z)\right) .
$$


An explicit expression for $\psi_{h}$ has been determined by Panny and Prodinger in (15, Theorem $2.2)$ :

$$
\psi_{h}(w)=\frac{\left(1+v^{2}\right)\left(1-v^{h+1}\right)^{2}}{(1-v)^{2}\left(1+v^{2 h+2}\right)}
$$

where $w=v /\left(1+v^{2}\right)$. By the definition of $H_{h}(z)$ it is clear that the relation between $z$ and $v$ is set by equation (11). Then it is just a matter of computation to obtain

$$
\begin{aligned}
H_{h}(z) & =\frac{\left(1+6 v^{2}+v^{4}\right)\left(1-v^{h+1}\right)^{2}}{(1-v)^{4}\left(1+v^{2(h+1)}\right)}, \\
\frac{1}{1-z} & =\frac{1+6 v^{2}+v^{4}}{(1-v)^{4}},
\end{aligned}
$$

whence, substituting in (2), the claim.

\section{Lemma 4.3.}

$$
\sum_{h \geq 1} \frac{v^{h}}{1+v^{2 h}} \stackrel{v \rightarrow 1^{-}}{\sim} \frac{\pi}{4(1-v)} .
$$

Proof. The proof is quite standard, we report it here for completeness. Put $v=e^{-t}, g(w)=$ $e^{-w} /\left(1+e^{-2 w}\right)$ and consider

$$
f(t):=\sum_{h \geq 1} \frac{e^{-h t}}{1+e^{-2 h t}}=\sum_{h \geq 1} g(h t) .
$$

The Mellin transform of $f$ is:

$$
\begin{aligned}
f^{*}(s) & =\int_{0}^{\infty} \sum_{h \geq 1} g(h t) t^{s-1} d t \\
& =\zeta(s) \int_{0}^{\infty} \sum_{\lambda \geq 0}(-1)^{\lambda} e^{-(2 \lambda+1) w} w^{s-1} d w
\end{aligned}
$$

where $\zeta(s)=\sum_{h \geq 1} h^{-s}$ is the Riemann zeta function. The knowledge of the behaviour of $f^{*}(s)$ in a neighbourhood of 1 , will give us the behaviour of $f(t)$ in a neighbourhood of 0 . Substitute $y=(2 \lambda+1) w$ to obtain

$$
f^{*}(s)=\zeta(s) \kappa(s) \Gamma(s),
$$

where $\kappa(s)=\sum_{\lambda \geq 0} \frac{(-1)^{\lambda}}{(1+2 \lambda)^{s}}$, and $\Gamma(s)=\int_{0}^{\infty} e^{-y} y^{s-1} d y$ is the gamma function. Since as $s \rightarrow 1^{+}$

$$
\begin{gathered}
\zeta(s)=\frac{1}{s-1}+O(1), \\
\Gamma(s)=1+O(s-1),
\end{gathered}
$$

we are left with the computation of the asymptotic behaviour of $\kappa(s)$. We may write

$$
\kappa(s)=\frac{1}{4^{s}}(\zeta(s, 1 / 4)-\zeta(s, 3 / 4)),
$$


where $\zeta(s, a)=\sum_{h \geq 0}(a+h)^{-s}$ is the Hurwitz zeta function. Thus using the expansion of $\zeta(s, a)$ for $s$ close to 1 (see (20, Formula 13.21)) we obtain

$$
\kappa(s)=\frac{\pi}{4}+O(s-1) .
$$

Hence we get

$$
f^{*}(s) \stackrel{s \rightarrow 1^{+}}{\sim} \frac{\pi}{4(s-1)}
$$

Applying (6, Theorem 1, p.115),

$$
f(t) \stackrel{t \rightarrow 0^{+}}{\sim} \frac{\pi}{4 t}
$$

which, substituting $t=-\log (1-(1-v))$, gives the claim.

\section{Theorem 4.4.}

$$
\mathbb{E}\left[D_{n}^{x}\right] \stackrel{n \rightarrow \infty}{\sim} \frac{2^{-7 / 4} \pi}{\Gamma(5 / 4)} n^{1 / 4}
$$

Proof. By Proposition 4.2 and Lemma 4.3, it is clear that

$$
\sum_{n \geq 0} \mathbb{E}\left[D_{n}^{x}\right] z^{n} \stackrel{v \rightarrow 1^{-}}{\sim} \frac{4 \pi}{(1-v)^{5}} .
$$

Choosing the solution $v$ of equation (11) which is smaller than 1 when $z$ is smaller than 1 , and substituting it in (3) we obtain

$$
\sum_{n=0}^{\infty} \mathbb{E}\left[D_{n}^{x}\right] z^{n} \stackrel{z \rightarrow 1^{-}}{\sim} \frac{2^{-7 / 4} \pi}{(1-z)^{5 / 4}},
$$

and (by (9, Corollary 2)) the claim.

Recalling Theorem 2.2] and the inequalities

$$
D_{n}^{y} \leq \max _{0 \leq i \leq n}\left\|S_{i}\right\|_{\infty} \leq \max _{0 \leq i \leq n}\left\|S_{i}\right\|_{1} \leq D_{n}^{x}+D_{n}^{y}
$$

the following corollary is obvious.

Corollary 4.5. On $\mathbf{C}_{2}$, both $\mathbb{E}\left[D_{n}\left(\|\cdot\|_{1}\right)\right]$ and $\mathbb{E}\left[D_{n}\left(\|\cdot\|_{\infty}\right)\right]$, as $n$ goes to infinity, are asymptotic to $\sqrt{\pi / 2} n^{1 / 2}$.

\subsection{Walk dimension}

The maximal deviation of a random walk is linked to the first exit time from a ball of radius $k$. Indeed if we put $T_{k}=\min \left\{i: S_{i} \notin B_{k}\right\}$, where $B_{k}$ is the ball of radius $k$ centered in $(0,0)$, then

$$
\left(D_{n} \leq k\right)=\left(T_{k}>n\right) .
$$

Clearly the radius of the ball and $D_{n}$ must be computed with respect to the same norm on the graph. Usually one chooses the $\|\cdot\|_{1}$ norm (we write $T_{n}^{\infty}$ if $\|\cdot\|_{\infty}$ is considered). Indeed 
this choice will not affect the definition of the walk dimension, which, as we recalled in the introduction, is defined for a graph $G$ as $\delta_{w}(G)=\alpha$ if $\mathbb{E}\left[T_{n}\right]$ is of order $n^{\alpha}$ (and $T_{n}$ is computed with respect to the simple random walk on $G$ ).

By the results obtained so far it is clear that on $\mathbf{C}_{2}, \mathbb{E}\left[T_{n}\right]$ is of order $n^{2}$, since $\delta_{w}(\mathbb{Z})=2$. It is perhaps less obvious that the exact asymptotics are $n^{2}$ and that this also holds when dealing with $\mathbb{E}\left[T_{n}^{\infty}\right]$.

The first asymptotics are obtained by comparison with the simple random walk on $\mathbb{Z}$ : we sketch here the derivation of the asymptotics of $\mathbb{E}\left[T_{n}(\mathbb{Z})\right]$ via generating functions techniques. The behaviour of the latter sequence is well known, nevertheless we briefly report here a proof in order to give a hint as to how to prove $\mathbb{E}\left[T_{n}^{\infty}\right] \sim n^{2}$.

Proposition 4.6. $\mathbb{E}\left[T_{n}\right] \stackrel{n \rightarrow \infty}{\sim} n^{2}$ and $\mathbb{E}\left[T_{n}^{\infty}\right] \stackrel{n \rightarrow \infty}{\sim} n^{2}$.

Proof. We write

$$
\mathbb{E}\left[T_{n}\right]=\sum_{k \geq 0} \mathbb{P}\left(T_{n}>k\right)
$$

that is, $\mathbb{E}\left[T_{n}\right]=\Theta_{n}(1)$, where $\Theta_{n}(z)$ is the generating function of the sequence $\left\{\mathbb{P}\left(T_{n}>k\right)\right\}_{k \geq 0}=$ $\left\{\mathbb{P}\left(\max _{0 \leq i \leq k}\left\|S_{i}\right\|_{1} \leq n\right)\right\}_{k \geq 0}$. We claim that $\mathbb{P}\left(\max _{0 \leq i \leq k}\left\|S_{i}\right\|_{1} \leq n\right)=\mathbb{P}\left(\max _{0 \leq i \leq k}\left|\widetilde{S}_{i}\right| \leq n\right)$ where $\left\{\widetilde{S}_{i}\right\}_{i \geq 0}$ is a suitable representation of the simple random walk on $\mathbb{Z}$. Indeed it suffices to consider the map that associates to each trajectory on $\mathbf{C}_{2}$ a trajectory on $\mathbb{Z}$ which jumps from $j$ to $j+1$ whenever on $\mathbf{C}_{2}$ one of the two components increases and jumps from $j$ to $j-1$ otherwise (i.e. when one of the components decreases).

The generating function of the number of paths on $\mathbb{Z}$ of length $k$, with maximal deviation smaller or equal to $n$ is $\Psi_{n}(z)=\frac{\left(1+v^{2}\right)\left(1-v^{n+1}\right)^{2}}{(1-v)^{2}\left(1+v^{2 n+2}\right)}$ with the substitution $z=v /\left(1+v^{2}\right)$, which was obtained in Theorem 2.2 of (15). Thus

$$
\Theta_{n}(z)=\frac{\left(1+v^{2}\right)\left(1+v+\cdots+v^{n}\right)^{2}}{\left(1+v^{2 n+2}\right)}
$$

with the substitution $z=2 v /\left(1+v^{2}\right)$. For $z=v=1$ we get $\Theta_{n}(1)=(n+1)^{2}$.

The same methods used for $\mathbb{E}\left[T_{n}\right]$ apply to the evaluation of $\mathbb{E}\left[T_{n}^{\infty}\right]$ (one only has to carefully decompose the walk much in the spirit of what was done in Lemma 4.1). The proof of this last estimate is omitted.

Corollary 4.7. The walk dimension of $\mathbf{C}_{2}$ is equal to 2.

\section{Mean maximal span}

Theorem 5.1. $\mathbb{E}\left[M_{n}^{x}\right]^{n \rightarrow \infty} \frac{2^{1 / 4}}{\Gamma(5 / 4)} n^{1 / 4}$.

Proof. Let $m_{n}^{x}=\max \left\{S_{i}^{x}: 0 \leq i \leq n\right\}$. Then it is clear that $\mathbb{E}\left[M_{n}^{x}\right]=2 \mathbb{E}\left[m_{n}^{x}\right]$. Our first aim is to compute the generating function of $\left\{\mathbb{P}\left(m_{n}^{x} \leq h\right)\right\}_{n \geq 0}$, which we denote by $\widetilde{\Psi}_{h}(z)$. Then

$$
\widetilde{\Psi}_{h}(z)=\lim _{k \rightarrow \infty} \sum_{l=-k}^{h} \widetilde{\Psi}_{h, k ; l}(z) \cdot E(z),
$$


where $\widetilde{\Psi}_{h, k ; l}(z)$ is the generating function of the probabilities of the $n$-step paths such that $-k \leq S_{i}^{x} \leq h$ for $0 \leq i \leq n, S_{n}=(l, 0)$ and $S_{n-1}^{x} \neq l$, while $E(z)$ was defined and computed in Lemma 4.1. Let us note that $\widetilde{\Psi}_{h, k ; l}(z)=\Psi_{h, k ; l}(z \widetilde{G}(0,0 \mid z) / 4)$, where $\Psi_{h, k ; l}(z)$ is the generating function of the number of the $n$-step paths on $\mathbb{Z}$ which stay in the interval between $-k$ and $h$, and end at $l$. The functions $\Psi_{h, k ; l}(w)$ are determined by the linear system used in (15, Theorem 4.1) to determine $\Psi_{h, k ; 0}(w)$. Thus

$$
\begin{aligned}
& \Psi_{h, k ; l}(w)=\frac{w^{l} a_{h-l-1}(w) a_{k-1}(w)}{a_{h+k}(w)}, \text { if } l \geq 0, \\
& \Psi_{h, k ; l}(w)=\frac{w^{-l} a_{h-1}(w) a_{k+l-1}(w)}{a_{h+k}(w)}, \text { if } l \leq-1 .
\end{aligned}
$$

Then we put $w=z \widetilde{G}(0,0 \mid z) / 4=v /\left(1+v^{2}\right)$ and we obtain that (compare with the proof of Proposition 4.21

$$
\begin{aligned}
\lim _{k \rightarrow \infty} \sum_{l=-k}^{h} \widetilde{\Psi}_{h, k ; l}(z) & =\frac{\left(1+v^{2}\right)\left(1-v^{h+1}\right)}{(1-v)^{2}}, \\
E(z) & =\frac{1+6 v^{2}+v^{4}}{\left(1+v^{2}\right)(1-v)^{2}}
\end{aligned}
$$

Then

$$
\begin{aligned}
\widetilde{\Psi}_{h}(z) & =\frac{\left(1+6 v^{2}+v^{4}\right)\left(1-v^{h+1}\right)}{(1-v)^{4}} \\
\sum_{n=0}^{\infty} \mathbb{E}\left[m_{n}^{x}\right] z^{n} & =\frac{1+6 v^{2}+v^{4}}{(1-v)^{4}} \sum_{h \geq 0} v^{h+1} \stackrel{z \rightarrow 1^{-}}{\sim} \frac{1}{2^{3 / 4}(1-z)^{5 / 4}}
\end{aligned}
$$

whence $\mathbb{E}\left[m_{n}^{x}\right] \stackrel{n \rightarrow \infty}{\sim} 2^{-3 / 4} n^{1 / 4} / \Gamma(5 / 4)$ and we are done.

Corollary 5.2. On $\mathbf{C}_{2}$, both $\mathbb{E}\left[M_{n}\left(\|\cdot\|_{1}\right)\right]$ and $\mathbb{E}\left[M_{n}\left(\|\cdot\|_{\infty}\right)\right]$, as $n$ goes to infinity, are asymptotic to $\sqrt{8 / \pi} n^{1 / 2}$.

\section{$6 \quad$ Scaling limits}

In the preceding sections we have seen that the expected values of the distances (with various meanings of this word) reached in $n$ steps are of order $n^{1 / 4}$ for the horizontal direction and of order $n^{1 / 2}$ for the vertical direction. These results lead us to a natural question: what is the asymptotic behaviour of the process where the horizontal component of the position after $n$ steps is divided by $n^{1 / 4}$ and the vertical component is divided by $n^{1 / 2}$ ? Of course we have to make this question more precise.

In order to study the scaling of the process we choose a suitable realization for the sequence $\left\{S_{n}\right\}_{n \geq 0}$ : let $X=\left\{X_{n}\right\}_{n \geq 0}$ be a sequence of random variables representing a simple random walk on $\mathbb{Z}$, and $Y=\left\{Y_{n}\right\}_{n \geq 0}$ be a sequence representing the random walk on $\mathbb{Z}$ moving according 
to Figure 2. Choose $X$ and $Y$ to be independent and let $X_{0}=Y_{0} \equiv 0$ almost surely. Moreover, let $L_{k}$ be the number of loops performed by $Y$ up to time $k$, that is

$$
L_{k}=\sum_{i=0}^{k-1} \mathbb{1}_{Y_{i}=0, Y_{i+1}=0} .
$$

Clearly, $S_{n}=\left(X_{L_{n}}, Y_{n}\right)$ is a realization of the position of the simple random walker on $\mathbf{C}_{2}$ at time $n$. We are now able to define, by linear interpolation of the three discrete time processes $X, Y$ and $L$, a continuous time process $\left(X_{L_{n t}}, Y_{n t}\right)$.

\section{Theorem 6.1.}

$$
\left(\frac{X_{L_{n t}}}{\sqrt[4]{n}}, \frac{Y_{n t}}{\sqrt{n}}\right)_{t \geq 0} \stackrel{\text { Law }}{\longrightarrow}\left(W_{L_{t}^{0}(B)}, B_{t}\right)_{t \geq 0}
$$

where $W$ and $B$ are two independent Brownian motions and $L_{t}^{0}(B)$ is the local time at 0 of $B$.

The theorem will be a consequence of Proposition 6.4 and Proposition 6.5] We introduce the following notion of convergence of stochastic processes (see Definition 2.2 of (5)).

Definition 6.2. A sequence of $\mathbb{R}^{k}$-valued stochastic processes $\left(Z_{t}^{n} ; t \geq 0\right)_{n \geq 0}$ converges to a process $\left(Z_{t} ; t \geq 0\right)$ in probability uniformly on compact intervals if for all $t \geq 0$, as $n \rightarrow \infty$

$$
\sup _{s \leq t}\left\|Z_{s}^{n}-Z_{s}\right\| \stackrel{\mathbb{P}}{\longrightarrow} 0
$$

where $\|\cdot\|$ is a norm on $\mathbb{R}^{k}$ (for instance $\|\cdot\|_{1}$ ). We will briefly write

$$
\left(Z_{t}^{n} ; t \geq 0\right) \stackrel{U . P}{\longrightarrow}\left(Z_{t} ; t \geq 0\right) .
$$

Since U.P. convergence of a vector is equivalent to U.P. convergence of its components and implies convergence in distribution, in order to prove Theorem 6.1 it will suffice to prove that each component in (5) U.P. converges to the corresponding limit.

The main idea is that $Y$ and $L$ are not much different from, respectively, a simple random walk $Y^{\prime}$ on $\mathbb{Z}$ and the process $L^{\prime}$ which counts the visits of $Y^{\prime}$ to 0 . There is a natural correspondence between $Y$ and $Y^{\prime}$, so let us define $Y^{\prime}$ and some other auxiliary variables which will be needed in the sequel. Given $Y$, let $l$ and $N$ be the processes which respectively count its returns to 0 (not including the loops and counting time 0 as the first "return") and the time spent not looping at 0 . Namely, let $l_{0}=1$, and $l_{k}=1+\sum_{i=0}^{k-1} \mathbb{1}_{Y_{i+1}=0, Y_{i} \neq 0}$ and $N_{k}=\sum_{i=0}^{k-1} \mathbb{1}_{Y_{i} \neq Y_{i+1}}$ for $k \geq 1$. Clearly $N_{k}=k-L_{k}$. Moreover, note that for $k \geq 1$,

$$
\sum_{i=0}^{l_{k}-1} \tau_{i} \leq L_{k} \leq \sum_{i=0}^{l_{k}} \tau_{i}
$$

where $\tau=\left\{\tau_{i}\right\}_{i \geq 0}$ is a suitable sequence of iid random variables with geometric distribution of parameter $1 / 2$. Now define a simple random walk $Y^{\prime}$ on $\mathbb{Z}$ by $Y_{n}=Y_{N_{n}}^{\prime}$. Then $L_{k}^{\prime}=\sum_{i=0}^{k} \mathbb{1}_{Y_{i}^{\prime}=0}$ ( $L^{\prime}$ counts the visits at 0 or, equivalently, the returns to 0 ). We note that $l_{n}=L_{N_{n}}^{\prime}$ and $0 \leq l_{n} \leq L_{n}^{\prime}$. We first prove a property of $L$. 


\section{Lemma 6.3.}

$$
\frac{L_{n}}{\sqrt{n}} \stackrel{\text { Law }}{\longrightarrow}|\mathcal{N}(0,1)|
$$

Proof. The main ideas of the proof are the facts that $L_{n}^{\prime} / \sqrt{n} \rightarrow|\mathcal{N}(0,1)|$ and that $N_{n}$ is not much different from $n$. Indeed one can easily prove the first fact (for the distribution of $\left(L_{2 n}^{\prime}-1\right)$ see (7), Chapter III, Exercise 10)). By (6), the claim is a consequence of

$$
\frac{\sum_{i=0}^{l_{n}} \tau_{i}}{\sqrt{n}} \stackrel{\operatorname{Law}}{\rightarrow}|\mathcal{N}(0,1)| \text {. }
$$

By the strong law of large numbers and Slutzky's theorem,

$$
\frac{\sum_{i=0}^{L_{n}^{\prime}} \tau_{i}}{\sqrt{n}}=\frac{\sum_{i=0}^{L_{n}^{\prime}} \tau_{i}}{L_{n}^{\prime}} \cdot \frac{L_{n}^{\prime}}{\sqrt{n}} \stackrel{\operatorname{Law}}{\rightarrow}|\mathcal{N}(0,1)| .
$$

Then (7) will follow once we show that $\sum_{i=0}^{l_{n}} \tau_{i} / \sum_{i=0}^{L_{n}^{\prime}} \tau_{i} \stackrel{\mathbb{P}}{\rightarrow} 1$. Indeed note that

$$
L_{n}^{\prime}=L_{N_{n}}^{\prime}+R_{n}^{\prime}=l_{n}+R_{n}^{\prime},
$$

where $R_{n}^{\prime}$ is the number of visits to 0 of $Y^{\prime}$ between time $N_{n}$ and time $n$. Let $T_{n}$ be the time $Y^{\prime}$ first visits 0 after time $N_{n}$, and for any $j \geq 0$ let $L_{j}^{\prime \prime}$ be the number of visits to 0 before time $j$ of the random walk $Y_{m}^{\prime \prime}:=Y_{m+T_{n}}^{\prime}$. Clearly $L_{j}^{\prime \prime}$ is independent of $\left\{Y_{k}^{\prime}\right\}_{k=0}^{N_{n}}$ and has the same distribution of $L_{j}^{\prime}$. Then

$$
\frac{\sum_{i=0}^{l_{n}} \tau_{i}}{\sum_{i=0}^{L_{n}^{\prime}} \tau_{i}}=1-\frac{\sum_{i=l_{n}+1}^{L_{n}^{\prime}} \tau_{i}}{\sum_{i=0}^{L_{n}^{\prime}} \tau_{i}}=1-\frac{\sum_{i=1}^{R_{n}^{\prime}} \widetilde{\tau}_{i}}{\sum_{i=0}^{L_{n}^{\prime}} \tau_{i}}
$$

where $\widetilde{\tau}_{i}=\tau_{i+l_{n}}$ and $\sum_{i=1}^{R_{n}^{\prime}} \widetilde{\tau}_{i}$ is equal to zero if $L_{n}^{\prime}=l_{n}$. We are left with the proof that $\sum_{i=1}^{R_{n}^{\prime}} \widetilde{\tau}_{i} / \sum_{i=0}^{L_{n}^{\prime}} \tau_{i} \stackrel{\mathbb{P}}{\rightarrow} 0$. Since $0 \leq R_{n}^{\prime} \leq L_{n-N_{n}}^{\prime \prime}$, it suffices to prove that $\sum_{i=1}^{L_{n-N_{n}}^{\prime \prime}} \widetilde{\tau}_{i} / \sum_{i=0}^{L_{n}^{\prime}} \tau_{i} \stackrel{\mathbb{P}}{\rightarrow} 0$. Fix $\varepsilon>0$ : since $\sum_{i=0}^{L_{n}^{\prime}} \tau_{i} \geq n-N_{n}$ we have that

$$
\begin{aligned}
\mathbb{P}\left(\frac{\sum_{i=1}^{L_{n-N_{n}}^{\prime \prime}} \widetilde{\tau}_{i}}{\sum_{i=0}^{L_{n}^{\prime}} \tau_{i}}>\varepsilon\right) & \leq \sum_{k=1}^{n} \mathbb{P}\left(\frac{\sum_{i=1}^{L_{n-N_{n}}^{\prime \prime}} \widetilde{\tau}_{i}}{n-N_{n}}>\varepsilon, n-N_{n}=k\right) \\
& =\sum_{k=1}^{n} \mathbb{P}\left(\frac{\sum_{i=1}^{L_{k}^{\prime \prime}} \widetilde{\tau}_{i}}{k}>\varepsilon\right) \mathbb{P}\left(n-N_{n}=k\right) .
\end{aligned}
$$

Now fix $\delta>0$ and choose $M=M(\delta)$ such that $\mathbb{P}\left(\sum_{i=1}^{L_{k}^{\prime \prime}} \widetilde{\tau}_{i} / k>\varepsilon\right)<\delta$ for all $k \geq M$ (this is possible by the law of large numbers using the facts that $L^{\prime \prime}$ and $\widetilde{\tau}$ are independent and $\left.L_{k}^{\prime \prime} / k \stackrel{\mathbb{P}}{\rightarrow} 0\right)$. Then

$$
\sum_{k=1}^{n} \mathbb{P}\left(\frac{\sum_{i=1}^{L_{k}^{\prime \prime}} \widetilde{\tau}_{i}}{k}>\varepsilon\right) \mathbb{P}\left(n-N_{n}=k\right) \leq \mathbb{P}\left(n-N_{n}<M\right)+\delta .
$$

Since $n-N_{n} \stackrel{\mathbb{P}}{\rightarrow} \infty$ we are done. 


\section{Proposition 6.4.}

$$
\left(\frac{1}{\sqrt{n}} Y_{n t}, \frac{1}{\sqrt{n}} L_{n t}\right)_{t \geq 0} \stackrel{U . P}{\longrightarrow}\left(B_{t}, L_{t}^{0}(B)\right)_{t \geq 0}
$$

Proof. Consider the processes $Y^{\prime}$ and $L^{\prime}$ defined before Lemma 6.3 and by interpolation define the sequence of two-dimensional continuous time processes $\left(\left(\frac{1}{\sqrt{n}} Y_{n t}^{\prime}, \frac{1}{\sqrt{n}} L_{n t}^{\prime}\right) ; t \geq 0\right)_{n \geq 0}$. Then by Theorem 3.1 of (5) we have that

$$
\left(\frac{1}{\sqrt{n}} Y_{n t}^{\prime}, \frac{1}{\sqrt{n}} L_{n t}^{\prime}\right)_{t \geq 0} \stackrel{U . P}{\longrightarrow}\left(B_{t}, L_{t}^{0}(B)\right)_{t \geq 0}
$$

To prove our statement, it suffices to show that these two properties hold:

$$
\begin{aligned}
& (A):\left(\frac{1}{\sqrt{n}}\left(Y_{\lfloor n t\rfloor}-Y_{\lfloor n t\rfloor}^{\prime}\right)\right)_{t \geq 0} \stackrel{U . P .}{\longrightarrow} 0 \\
& (B):\left(\frac{1}{\sqrt{n}}\left(L_{\lfloor n t\rfloor}-L_{\lfloor n t\rfloor}^{\prime}\right)\right)_{t \geq 0} \stackrel{U . P}{\longrightarrow} 0 .
\end{aligned}
$$

Note that $Y^{\prime}$ is the sum of iid increments $\xi_{i}$ such that $\mathbb{P}\left(\xi_{i}= \pm 1\right)=1 / 2$, hence

$$
\begin{aligned}
Y_{\lfloor n t\rfloor} & =Y_{\lfloor n t\rfloor-L_{\lfloor n t\rfloor}^{\prime}}^{\prime}=\sum_{i=1}^{\lfloor n t\rfloor-L_{\lfloor n t\rfloor}} \xi_{i} \\
& =\sum_{i=1}^{\lfloor n t\rfloor} \xi_{i}-\sum_{i=\lfloor n t\rfloor-L_{\lfloor n t\rfloor}+1}^{\lfloor n t\rfloor} \xi_{i}=Y_{\lfloor n t\rfloor}^{\prime}-\sum_{i=1}^{L_{\lfloor n t\rfloor}} \tilde{\xi}_{i},
\end{aligned}
$$

where $\tilde{\xi}_{i}=\xi_{\lfloor n t\rfloor-L_{\lfloor n t\rfloor}+i}\left(\right.$ and $\sum_{i=1}^{L_{\lfloor n t\rfloor}} \tilde{\xi}_{i}=0$ if $\left.L_{\lfloor n t\rfloor}=0\right)$. Thus $Y_{\lfloor n t\rfloor}-Y_{\lfloor n t\rfloor}^{\prime}=\sum_{i=1}^{L_{\lfloor n t\rfloor}} \tilde{\xi}_{i}$. Then (A) follows from

$$
\sup _{s \leq t}\left|\frac{1}{\sqrt{n}} \sum_{i=1}^{L_{\lfloor n s\rfloor}} \tilde{\xi}_{i}\right| \stackrel{\mathbb{P}}{\longrightarrow} 0 .
$$

Indeed

$$
\sup _{s \leq t}\left|\sum_{i=1}^{L_{\lfloor n s\rfloor}} \tilde{\xi}_{i}\right| \leq \max _{k \leq L_{\lfloor n t\rfloor}}\left|\sum_{i=1}^{k} \tilde{\xi}_{i}\right|,
$$

and if we denote by $M_{n}=\max _{k \leq n} \sum_{i=1}^{k} \tilde{\xi}_{i}$ and by $m_{n}=\min _{k \leq n} \sum_{i=1}^{k} \tilde{\xi}_{i}$, clearly $M_{n}$ and $-m_{n}$ are identically distributed, and $\max _{k \leq L}{ }_{\lfloor n t\rfloor}\left|\sum_{i=1}^{k} \tilde{\xi}_{i}\right|=\max \left\{M_{L_{\lfloor n t\rfloor}},-m_{L_{\lfloor n t\rfloor}}\right\}$ Hence to prove (9) it suffices to show that

$$
\frac{1}{\sqrt{n}} M_{L_{\lfloor n t\rfloor}} \stackrel{\mathbb{P}}{\longrightarrow} 0
$$


The distribution of $M_{n}$ is well known (see (7, Chapter III.7)), and it is easy to show that $M_{n} / \sqrt{n} \stackrel{\text { Law }}{\rightarrow}|\mathcal{N}(0,1)|$. Noting that $L_{\lfloor n t\rfloor}$ is independent of $M_{k}$, we have that

$$
\begin{aligned}
\mathbb{P}\left(M_{L_{\lfloor n t\rfloor}}>\varepsilon \sqrt{n}\right) & =\sum_{k=0}^{\lfloor n t\rfloor} \mathbb{P}\left(M_{k}>\varepsilon \sqrt{n}\right) \mathbb{P}\left(L_{\lfloor n t\rfloor}=k\right) \\
& \leq \sum_{\varepsilon \sqrt{n}<k \leq \alpha \sqrt{\lfloor n t\rfloor}} \mathbb{P}\left(M_{k}>\varepsilon \sqrt{n}\right) \mathbb{P}\left(L_{\lfloor n t\rfloor}=k\right)+\sum_{k>\alpha \sqrt{\lfloor n t\rfloor}} \mathbb{P}\left(L_{\lfloor n t\rfloor}=k\right) \\
& \leq \mathbb{P}\left(M_{\lfloor\alpha \sqrt{\lfloor n t\rfloor\rfloor}}>\varepsilon \sqrt{n}\right)+\mathbb{P}\left(L_{\lfloor n t\rfloor}>\alpha \sqrt{\lfloor n t\rfloor}\right) .
\end{aligned}
$$

By Lemma 6.3. for any positive $\varepsilon^{\prime}$ and $t$ there exist $\alpha$ and $n^{\prime}$ such that $\mathbb{P}\left(L_{\lfloor n t\rfloor}>\alpha \sqrt{\lfloor n t\rfloor}\right)<\varepsilon^{\prime}$ for all $n \geq n^{\prime}$. On the other hand for any given $\varepsilon, \alpha$

$$
\mathbb{P}\left(\frac{M_{\lfloor\alpha \sqrt{\lfloor n t\rfloor}}}{\sqrt{\alpha} \sqrt[4]{\lfloor n t\rfloor}}>\frac{\varepsilon \sqrt[4]{n}}{\sqrt{\alpha} \sqrt[4]{t}}\right) \stackrel{n \rightarrow \infty}{\longrightarrow} 0
$$

whence $(A)$ is proven.

Now, let us address to $(B)$. We first note that we may consider a mapping between the number of steps taken by $Y^{\prime}$ and the ones taken by $Y$. Indeed when $Y^{\prime}$ has taken $\lfloor n t\rfloor$ steps, then $Y$ has taken $\lfloor n t\rfloor+\sum_{i \leq L_{\lfloor n t\rfloor}^{\prime}} \tau_{i}$ steps (that is, if $Y_{\lfloor n t\rfloor}^{\prime}=0$ we decide to count for $Y$ all the loops it performs after this last return to 0$)$. Let us write

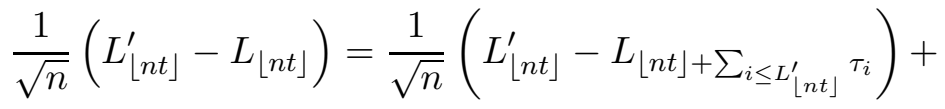

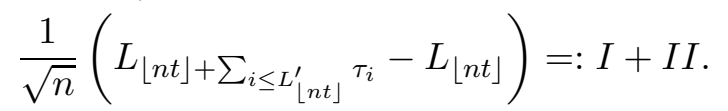

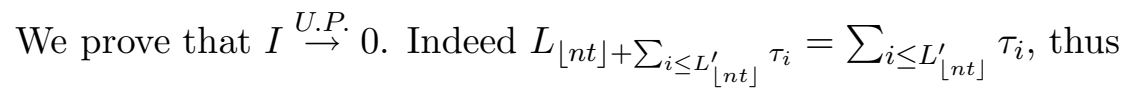

$$
\sup _{s \leq t}\left|L_{\lfloor n s\rfloor}^{\prime}-\sum_{i \leq L_{\lfloor n s\rfloor}^{\prime}} \tau_{i}\right|=\sup _{s \leq t}\left|\sum_{i \leq L_{\lfloor n s\rfloor}^{\prime}}\left(1-\tau_{i}\right)\right| \leq \max _{k \leq L_{\lfloor n t\rfloor}^{\prime}}\left|\sum_{i \leq k}\left(1-\tau_{i}\right)\right| .
$$

Now choose $\delta>0$. By independence of $L^{\prime}$ and $\tau$ we have that the probability that $\max _{k \leq L_{\lfloor n t\rfloor}^{\prime}}\left|\sum_{i \leq k}\left(1-\tau_{i}\right)\right|$ is larger than $\varepsilon \sqrt{n}$ is bounded by

$$
\mathbb{P}\left(L_{\lfloor n t\rfloor}^{\prime}>\alpha \sqrt{\lfloor n t\rfloor}\right)+\sum_{j \leq \alpha \sqrt{\lfloor n t\rfloor}} \mathbb{P}\left(\max _{k \leq j}\left|\sum_{i \leq k}\left(1-\tau_{i}\right)\right|>\varepsilon \sqrt{n}\right) \mathbb{P}\left(L_{\lfloor n t\rfloor}^{\prime}=j\right) .
$$

The first term is smaller than $\delta$ if $\alpha$ and $n$ are sufficiently large. As for the second term, it is clearly less or equal to

$$
\mathbb{P}\left(\max _{k \leq \alpha \sqrt{\lfloor n t\rfloor}}\left|\sum_{i \leq k}\left(1-\tau_{i}\right)\right|>\varepsilon \sqrt{n}\right) .
$$


Observe that, by the law of large numbers, for any positive $\varepsilon^{\prime}$ and $\delta$ there exists $k_{0}=k_{0}\left(\varepsilon^{\prime}, \delta\right)$ such that for all $k \geq k_{0}$

$$
\mathbb{P}\left(\left|\frac{\sum_{i \leq k}\left(1-\tau_{i}\right)}{k}\right|<\varepsilon^{\prime}\right) \geq 1-\delta .
$$

Hence (11) is less or equal to

$$
\mathbb{P}\left(\max _{k \leq k_{0}}\left|\sum_{i \leq k}\left(1-\tau_{i}\right)\right|>\varepsilon \sqrt{n}\right)+\mathbb{P}\left(\max _{k_{0} \leq k \leq \alpha \sqrt{\lfloor n t\rfloor}}\left|\sum_{i \leq k}\left(1-\tau_{i}\right)\right|>\varepsilon \sqrt{n}\right) .
$$

The first term clearly tends to 0 as $n$ grows to infinity, while the second term is not larger than

$$
\delta+\mathbb{P}\left(\max _{k_{0} \leq k \leq \alpha \sqrt{\lfloor n t\rfloor}}\left|\sum_{i \leq k}\left(1-\tau_{i}\right)\right|>\varepsilon \sqrt{n},\left|\frac{\sum_{i \leq k}\left(1-\tau_{i}\right)}{k}\right|<\varepsilon^{\prime} \quad \forall k \geq k_{0}\right) .
$$

But if $\left|\sum_{i \leq k}\left(1-\tau_{i}\right)\right| / k<\varepsilon^{\prime}$ for all $k \geq k_{0}$, then

$$
\sup _{k_{0} \leq k \leq \alpha \sqrt{\lfloor n t\rfloor}}\left|\sum_{i \leq k}\left(1-\tau_{i}\right)\right|<\alpha \varepsilon^{\prime} \sqrt{\lfloor n t\rfloor},
$$

which is smaller than $\varepsilon \sqrt{n}$ if $\varepsilon^{\prime}$ is sufficiently small. This proves that $I \stackrel{\text { U.P. }}{\rightarrow} 0$.

We now prove that $I I \stackrel{U . P .}{\rightarrow} 0$. Indeed

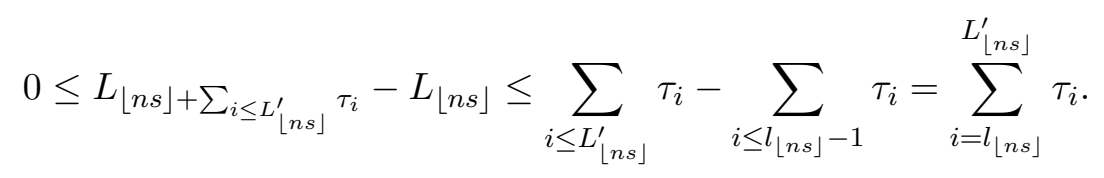

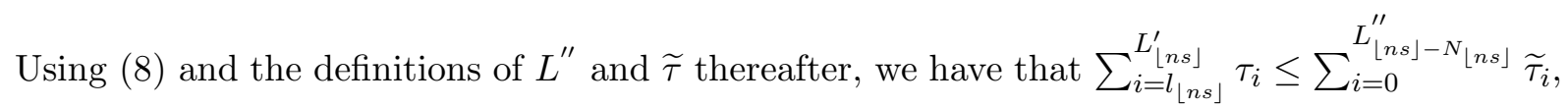
and for any positive $\varepsilon^{\prime}$,

$$
\sup _{s \leq t} \sum_{i=0}^{L_{\lfloor n s\rfloor-N\lfloor n s\rfloor}^{\prime \prime}} \tilde{\tau}_{i}=\max \left\{\sup _{s \leq \varepsilon^{\prime}} \sum_{i=0}^{L_{\lfloor n s\rfloor-N\lfloor n s\rfloor}^{\prime \prime}} \tilde{\tau}_{i} \sup _{\varepsilon^{\prime}<s \leq t} \sum_{i=0}^{L_{\lfloor n s\rfloor-N\lfloor n s\rfloor}^{\prime \prime}} \tilde{\tau}_{i}\right\}=: \max (C, D) .
$$

Choose $\delta>0$. Let us show that $\mathbb{P}(C>\varepsilon \sqrt{n})<\delta$ if $\varepsilon^{\prime}$ is sufficiently small and $n$ sufficiently large. Indeed

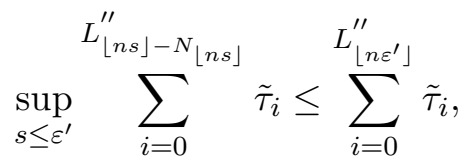

and by independence of $L_{\left\lfloor n \varepsilon^{\prime}\right\rfloor}^{\prime \prime}$ and $\tilde{\tau}$,

$$
\mathbb{P}\left(\sum_{i \leq L_{\left\lfloor n \varepsilon^{\prime}\right\rfloor}^{\prime \prime}} \tilde{\tau}_{i}>\varepsilon \sqrt{n}\right) \leq \mathbb{P}\left(L_{\left\lfloor n \varepsilon^{\prime}\right\rfloor}^{\prime \prime}>\alpha \sqrt{\left\lfloor n \varepsilon^{\prime}\right\rfloor}\right)+\mathbb{P}\left(\sum_{i \leq \alpha \sqrt{\left\lfloor n \varepsilon^{\prime}\right\rfloor}} \tilde{\tau}_{i}>\varepsilon \sqrt{n}\right)
$$


Choose $\alpha$ such that $\mathbb{P}\left(L_{\left\lfloor n \varepsilon^{\prime}\right\rfloor}^{\prime \prime}>\alpha \sqrt{\left\lfloor n \varepsilon^{\prime}\right\rfloor}\right)<\delta$ for $n$ sufficiently large, and then $\varepsilon^{\prime}$ sufficiently small such that $\mathbb{P}\left(\sum_{i \leq \alpha \sqrt{\left\lfloor n \varepsilon^{\prime}\right\rfloor}} \widetilde{\tau}_{i}>\varepsilon \sqrt{n}\right)<\delta$.

Now keep $\varepsilon^{\prime}$ fixed. In order to prove that $\mathbb{P}(D>\varepsilon \sqrt{n})<3 \delta$ for $n$ sufficiently large, we observe that for any positive $\varepsilon^{\prime \prime}$ there exists $\bar{n}$ such that for all $n \geq \bar{n}, \mathbb{P}\left(N_{n} \geq n\left(1-\varepsilon^{\prime \prime}\right)\right)$ is larger than $1-\delta$. Then for all $n$ such that $\left\lfloor n \varepsilon^{\prime}\right\rfloor \geq \bar{n}$, $\sup _{\varepsilon^{\prime}<s \leq t}\left(L_{\lfloor n s\rfloor-N_{\lfloor n s\rfloor}}^{\prime \prime}\right) \leq L_{\left\lfloor n t \varepsilon^{\prime \prime}\right\rfloor}^{\prime \prime}$ with probability larger than $1-\delta$. Hence, as in (12) we have that, for $\alpha$ and $n$ sufficiently large and $\varepsilon^{\prime \prime}$ sufficiently small.

$$
\begin{aligned}
\mathbb{P}(D>\varepsilon \sqrt{n}) & \leq \delta+\mathbb{P}\left(\sum_{i \leq L_{\left\lfloor n t \varepsilon^{\prime \prime}\right\rfloor}^{\prime \prime}} \tilde{\tau}_{i}>\varepsilon \sqrt{n}\right) \\
& \leq \delta+\mathbb{P}\left(L_{\left\lfloor n t \varepsilon^{\prime \prime}\right\rfloor}^{\prime \prime}>\alpha \sqrt{\left\lfloor n t \varepsilon^{\prime \prime}\right\rfloor}\right)+\mathbb{P}\left(\sum_{i \leq \alpha \sqrt{\left\lfloor n t \varepsilon^{\prime \prime}\right\rfloor}} \tilde{\tau}_{i}>\varepsilon \sqrt{n}\right)<3 \delta .
\end{aligned}
$$

\section{Proposition 6.5.}

$$
\left(\frac{X_{L_{n t}}}{\sqrt[4]{n}}\right)_{t \geq 0} \stackrel{U . P}{\longrightarrow}\left(W_{L_{t}^{0}(B)}\right)_{t \geq 0}
$$

Proof. Clearly the statement may be rephrased as

$$
\left(\frac{X_{L_{n^{2} t}}}{\sqrt{n}}\right)_{t \geq 0} \stackrel{U . P}{\longrightarrow}\left(W_{L_{t}^{0}(B)}\right)_{t \geq 0}
$$

Note that (writing $L_{s}^{0}$ instead of $L_{s}^{0}(B)$ )

$$
\sup _{s \leq t}\left|\frac{X_{L_{n^{2} s}}}{\sqrt{n}}-W_{L_{s}^{0}}\right| \leq \sup _{s \leq t}\left|\frac{X_{L_{n^{2} s}}-X_{n L_{s}^{0}}}{\sqrt{n}}\right|+\sup _{s \leq t}\left|\frac{X_{n L_{s}^{0}}}{\sqrt{n}}-W_{L_{s}^{0}}\right|=(A)+(B) .
$$

We have to show that $(A)$ and $(B) \stackrel{\text { U.P. }}{\rightarrow} 0$ : it suffices to prove the statement for $L_{\left\lfloor n^{2} s\right\rfloor}$ and $\left\lfloor n L_{s}^{0}\right\rfloor$ instead of $L_{n^{2} s}$ and $n L_{s}^{0}$ respectively. Represent $X_{k}=\sum_{1}^{k} \widetilde{\eta}_{i}$, where $\widetilde{\eta}=\left\{\widetilde{\eta}_{i}\right\}_{i \geq 1}$ is an iid family such that $\mathbb{P}\left(\widetilde{\eta}_{i}= \pm 1\right)=1 / 2$, then

$$
\sup _{s \leq t}\left|X_{L_{\left\lfloor n n^{2}\right\rfloor}}-X_{\left\lfloor n L_{s}^{0}\right\rfloor}\right|=\sup _{s \leq t}\left|\sum_{i=1}^{n \Delta_{n, s}} \eta_{i}\right|,
$$

where $n \Delta_{n, s}:=\left|L_{\left\lfloor n^{2} s\right\rfloor}-\left\lfloor n L_{s}^{0}\right\rfloor\right|$ and $\eta_{i}=\widetilde{\eta}_{\min \left\{L_{\left\lfloor n^{2} s\right\rfloor},\left\lfloor n L_{s}^{0}\right\rfloor\right\}+i}$. Note that $\left(\Delta_{n, t}\right)_{t \geq 0} \stackrel{U . P .}{\rightarrow} 0$, indeed

$$
\Delta_{n, t} \leq\left|\frac{L_{\left\lfloor n^{2} t\right\rfloor}}{n}-L_{t}^{0}\right|+\left|L_{t}^{0}-\frac{\left\lfloor n L_{t}^{0}\right\rfloor}{n}\right| \leq\left|\frac{L_{\left\lfloor n^{2} t\right\rfloor}}{n}-L_{t}^{0}\right|+\frac{1}{n},
$$


and both these summands tends U.P. to 0 (the first by Proposition 6.4). In order to show that

$$
\mathbb{P}\left(\sup _{s \leq t}\left|\sum_{i=1}^{n \Delta_{n, s}} \eta_{i}\right|>\varepsilon \sqrt{n}\right) \stackrel{n \rightarrow \infty}{\rightarrow} 0
$$

it suffices to prove that

$$
\mathbb{P}\left(\max _{k \leq \sup _{s \leq t}\left(n \Delta_{n, s}\right)} \sum_{i=1}^{k} \eta_{i}>\varepsilon \sqrt{n}\right) \stackrel{n \rightarrow \infty}{\rightarrow} 0 .
$$

Now put $M_{n}=\max _{k \leq n} \sum_{i=1}^{k} \eta_{i}$ and recall that $\left(n \Delta_{n, s}\right)_{n \geq 0, s \leq t}$ and $\eta$ are independent: this last probability may be written as

$$
\begin{aligned}
& \sum_{j=0}^{\infty} \mathbb{P}\left(M_{j}>\varepsilon \sqrt{n}\right) \mathbb{P}\left(\sup _{s \leq t}\left(n \Delta_{n, s}\right)=j\right) \\
& \leq \mathbb{P}\left(\sup _{s \leq t}\left(\Delta_{n, s}\right)>\alpha\right)+\mathbb{P}\left(M_{\lfloor n \alpha\rfloor}>\varepsilon \sqrt{n}\right) .
\end{aligned}
$$

For all positive $\alpha, \mathbb{P}\left(\sup _{s \leq t}\left(\Delta_{n, s}\right)>\alpha\right)$ can be made arbitrarily small if $n$ is picked large enough, while

$$
\mathbb{P}\left(M_{\lfloor n \alpha\rfloor}>\varepsilon \sqrt{n}\right) \stackrel{n \rightarrow \infty}{\rightarrow} \mathbb{P}\left(|\mathcal{N}(0,1)|>\frac{\varepsilon}{\sqrt{\alpha}}\right) .
$$

Thus if $\delta>0$ is fixed, by choosing $\alpha$ sufficiently small we get $\mathbb{P}\left(M_{\lfloor n \alpha\rfloor}>\varepsilon \sqrt{n}\right)<\delta$ if $n$ is sufficiently large. This proves $(A) \stackrel{\text { U.P. }}{\rightarrow} 0$.

Now note that $X_{n}$ and $L_{s}^{0}$ are independent, so we may think of $X_{\left\lfloor n L_{s}^{0}\right\rfloor}$ as defined on a product probability space $\Omega_{1} \otimes \Omega_{2}$ (that is $\left.X_{\left\lfloor n L_{s}^{0}\right\rfloor}\left(\omega_{1}, \omega_{2}\right)=X_{\left\lfloor n L_{s}^{0}\left(\omega_{2}\right)\right\rfloor}\left(\omega_{1}\right)\right)$. For any fixed $\omega_{2}$ we have that

$$
\mathbb{P}_{1}\left(\sup _{s \leq t}\left|\frac{X_{\left\lfloor n L_{s}^{0}\left(\omega_{2}\right)\right\rfloor}}{\sqrt{n}}-W_{L_{s}^{0}\left(\omega_{2}\right)}\right|>\varepsilon\right) \stackrel{n \rightarrow \infty}{\rightarrow} 0 .
$$

Indeed it is known (see (5) ) that $\mathbb{P}_{1}\left(\sup _{s \leq t}\left|\frac{X_{n s}}{\sqrt{n}}-W_{s}\right|>\varepsilon\right) \stackrel{n \rightarrow \infty}{\rightarrow} 0$, and since for any fixed $\omega_{2}$, as $s \uparrow t, L_{s}^{0}\left(\omega_{2}\right) \uparrow L_{t}^{0}\left(\omega_{2}\right)$,

$$
\sup _{s \leq t}\left|\frac{X_{\left\lfloor n L_{s}^{0}\left(\omega_{2}\right)\right\rfloor}}{\sqrt{n}}-W_{L_{s}^{0}\left(\omega_{2}\right)}\right| \leq \sup _{s \leq L_{t}^{0}\left(\omega_{2}\right)}\left|\frac{X_{\lfloor n s\rfloor}}{\sqrt{n}}-W_{s}\right|,
$$

whence (13). Thus, putting

$$
A_{n}:=\left\{\left(\omega_{1}, \omega_{2}\right): \sup _{s \leq t}\left|\frac{X_{\left\lfloor n L_{s}^{0}\left(\omega_{2}\right)\right\rfloor}\left(\omega_{1}\right)}{\sqrt{n}}-W_{L_{s}^{0}\left(\omega_{2}\right)}\left(\omega_{1}\right)\right|>\varepsilon\right\}
$$

we have that $\mathbb{P}_{1}\left(A_{n}\right) \rightarrow 0$, by Fubini and the dominated convergence theorem we get $\mathbb{P}_{1} \otimes$ $\mathbb{P}_{2}\left(A_{n}\right) \rightarrow 0$ and we are done. 


\section{Final remarks}

The inhomogeneity of $\mathbf{C}_{2}$ results in the difference between the behaviour of the horizontal and vertical components of the walk. Indeed while for any positive integer $d$ the expected value of the distance from the origin of the simple random walker on $\mathbb{Z}^{d}$, after $n$ steps, is of order $n^{1 / 2}$ (this may be easily proven via a conditioning argument, with respect to the proportions of time spent along each of the $d$ main directions), the expected value of the horizontal distance on $\mathbf{C}_{2}$ is of order $n^{1 / 4}$, that is on this direction we have a subdiffusive behaviour.

The difference between the two projections of the walk is remarkable also when one properly rescales the process as we did in Section 6. Indeed although both components scale to a Brownian motion, the horizontal one is much slower (its clock is the local time at 0 of the vertical component).

Finally, also the failure of the Einstein relation on $\mathbf{C}_{2}$ is due to the inhomogeneity of this particular graph. Indeed for strongly inhomogeneous graphs the growth exponent does not give an accurate description of the "fractal properties" of the graph (the assignment of the same $\delta_{f}$ of $\mathbb{Z}^{2}$ to $\mathbf{C}_{2}$ disregards the topology of the two structures). It seems that defining $\delta_{f}$ as the growth exponent of the graph makes sense only for homogeneous graphs (like $\mathbb{Z}^{d}$ ) or self-similar graphs, which have a clear fractal nature (like the Sierpiński graph). It is our opinion that the study of the sense in which a graph has a fractal nature and what is the proper definition of its fractal dimension should require further investigations.

\section{Acknowledgments}

I feel particularly indebted to Peter Grabner and Helmut Prodinger for raising the questions discussed in this paper and for the stimulating discussions during which they suggested the techniques used in Sections 3, 4 and 5. I would also like to thank Jean-Francois Le Gall for suggesting the limit of the rescaled process and giving me some precious hints.

\section{References}

[1] M.T. Barlow and R.F. Bass, (1999) Random walks on graphical Sierpiński carpets. Random walks and discrete potential theory (Cortona, 1997), 26-55, Sympos. Math., XXXIX, Cambridge Univ. Press, Cambridge. MR1802425

[2] E.A. Bender, (1974) Asymptotic methods in enumeration, SIAM Rev., 16, 485-515. MR0376369

[3] D. Bertacchi and F. Zucca, (2003) Uniform asymptotic estimates of transition probabilities on combs. J. Aust. Math. Soc. 75 no.3, 325-353. MR2015321

[4] D. Cassi and S. Regina, (1992) Random walks on $d$-dimensional comb lattices. Modern Phys. Lett. B 6 no.22, 1397-1403. MR1188155 
[5] A.S. Cherny, A.N. Shiryaev and M. Yor, (2002) Limit behaviour of the "horizontal-vertical" random walk and some extensions of the Donsker-Prokhorov invariance principle. Theory Probab. Appl. 47 no.3, 377-394. MR1975425

[6] G. Doetsch, (1956) Handbuch der Laplace-Transformation. Birkhäuser Verlag, BaselStuttgart. MR0344808

[7] W. Feller, (1968) An Introduction to Probability Theory and Its Applications, vol. I 3rd ed., John Wiley and Sons, New York. MR0228020

[8] W. Feller, (1971) An Introduction to Probability Theory and Its Applications, vol. II 2nd ed., John Wiley and Sons, New York. MR0270403

[9] P. Flajolet and A. Odlyzko, (1990) Singularity analysis of generating functions. SIAM J. Discrete Math. 3 no.2, 216-240. MR1039294

[10] P. Gerl, (1986) Natural spanning trees of $\mathbb{Z}^{d}$ are recurrent. Discrete Math. 61 no. 2-3, 333-336. MR0855341

[11] A. Grigor'yan and A. Telcs, (2001) Sub-Gaussian estimates of heat kernels on infinite graphs. Duke Math. J. 109 (2001) no.3, 451-510. MR1853353

[12] O.D. Jones, (1996) Transition probabilities for the simple random walk on the Sierpiński graph. Stochastic Process. Appl. 61, 4-69. MR1378848

[13] I. Karatzas and S. Shreve, (1991) Brownian motion and Stochastic Calculus, 2nd edition, Springer-Verlag, New York. MR1121940

[14] M. Krishnapur and Y. Peres, (2004) Recurrent graphs where two independent random walks collide finitely often. Electron. Comm. Prob. 9, 72-81. MR2081461

[15] W. Panny and H. Prodinger, (1985) The expected height of paths for several notions of height. Studia Sci. Math. Hungar. 20 no.1-4, 119-132. MR0886012

[16] A. Telcs, (1990) Spectra of graphs and fractal dimensions I. Probab. Theory Related Fields 85, 489-497. MR1061940

[17] A. Telcs, (1995) Spectra of graphs and fractal dimensions II., Probab. Theory Related Fields 8, 77-96. MR1308671

[18] A. Telcs, (2001) Local sub-Gaussian estimates on graphs, the strongly recurrent case. Electron. J. Probab. 6 no.22, 1-33. MR1873299

[19] S. Havlin and G.H. Weiss, (1986) Some properties of a random walk on a comb structure. Physica 134A, 474-482.

[20] E.T. Whittaker and G.N. Watson, (1962) A course of modern analysis. An introduction to the general theory of infinite processes and of analytic functions: with an account of the principal transcendental functions. Fourth edition. Cambridge Univ. Press, New York. MR0178117

[21] W. Woess, (2000) Random walks on infinite graphs and groups. Cambridge Tracts in Mathematics 138, Cambridge Univ. Press, Cambridge. MR1743100 